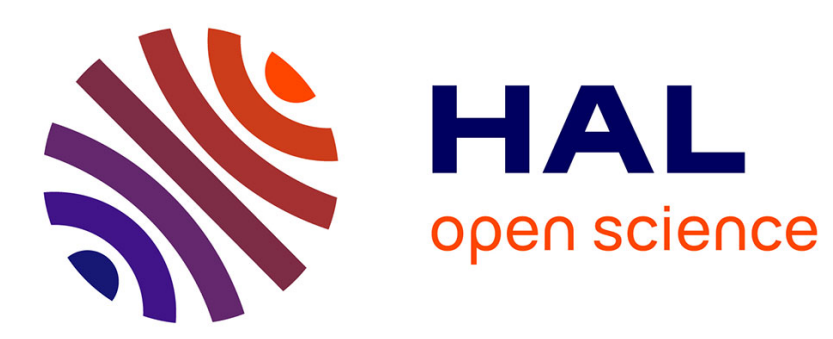

\title{
Génération des maillages pour la simulation par Eléments Finis de problèmes physiques
}

\author{
P.L. George
}

\section{To cite this version:}

P.L. George. Génération des maillages pour la simulation par Eléments Finis de problèmes physiques. Revue de Physique Appliquée, 1990, 25 (7), pp.567-581. 10.1051/rphysap:01990002507056700 . jpa00246222

\section{HAL Id: jpa-00246222 https://hal.science/jpa-00246222}

Submitted on 1 Jan 1990

HAL is a multi-disciplinary open access archive for the deposit and dissemination of scientific research documents, whether they are published or not. The documents may come from teaching and research institutions in France or abroad, or from public or private research centers.
L'archive ouverte pluridisciplinaire HAL, est destinée au dépôt et à la diffusion de documents scientifiques de niveau recherche, publiés ou non, émanant des établissements d'enseignement et de recherche français ou étrangers, des laboratoires publics ou privés. 


\title{
Génération des maillages pour la simulation par Eléments Finis de problèmes physiques
}

\author{
P. L. George \\ INRIA Domaine de Voluceau - Rocquencourt, BP 105, 78153 Le Chesnay, France
}

(Reçu le 27 octobre 1989, accepté le 22 février 1990)

\begin{abstract}
Résumé. - La simulation par la Méthode des Eléments Finis de problèmes physiques modélisés en termes d'équations aux dérivées partielles nécessite dans une première phase la construction d'un maillage du domaine de calcul. Cet article se propose de présenter différentes méthodes permettant la génération de tels maillages.
\end{abstract}

Abstract. - This paper describes some practical approaches to be used for the purpose of generation of meshes related to Finite Element computation of physical problems discretised in term of P.D.E.

\section{Introduction.}

La simulation numérique par la Méthode des Eléments Finis [6] de nombreux problèmes physiques formulés en termes d'équations aux dérivées partielles nécessite, dans une première étape, la construction d'un maillage du domaine de calcul.

Nous allons présenter la façon de procéder pour obtenir un tel maillage. Après avoir donné une définition possible de cette notion de maillage, nous allons introduire une méthodologie adaptée permettant de résoudre les problèmes rencontrés lors de sa conception.

Plusieurs parties seront développées qui présenteront :

- le concept de maillage et quelques structurations associées ;

- une méthode d'analyse des domaines à mailler en fonction, en particulier, de leur géométrie et des algorithmes disponibles;

tant en dimension 2 qu'en dimension 3 ;

- quelques méthodes de manipulation de maillages ;

- quelques logiciels ou progiciels de création de maillages.

\section{Notion de maillages.}

2.1 DÉFINITION FORMELLE. - Un maillage d'un domaine $\Omega$ est un ensemble $M$ d'éléments $E_{i}$ tel que : $\bar{\Omega}=\mathbf{M}=\bigcup \mathrm{E}_{i}$.
Les $\mathrm{E}_{i}$ peuvent être des segments, des triangles, des quadrangles en dimension 2 ou ces mêmes éléments et des tétraèdres, des pentaèdres et des hexaèdres en dimension 3 .

Les $\mathrm{E}_{i}$ doivent vérifier la condition suivante (voir Fig. 1):

- l'intersection de deux éléments $\mathrm{E}_{i}$ et $\mathrm{E}_{j}$ de $\mathrm{M}$ doit être soit vide, soit réduite à un point, une arête ou une face.

Cette définition revient à indiquer que $\mathbf{M}$ recouvre $\Omega$ de manière conforme, elle correspond à une approche essentiellement géométrique.

2.2 DÉfinition PRATIQUe. - Afin de pouvoir utiliser un maillage pour effectuer des calculs par la Méthode des Eléments Finis, il convient, à côté de l'aspect géométrique vu ci-dessus, de rajouter des informations de nature physique. Grâce à celles-ci la localisation des conditions limites et des matériaux ques des cas de charges, des blocages, des caractéristiques de ces matériaux, ... pourront être affectées.

\section{Structurations associées.}

Un maillage comporte donc des informations de type géométrique qui concernent la description du recouvrement du domaine considéré auxquelles s'ajoutent des informations de type physique liées à la nature des équations modélisant le problème traité.

Il est commode de définir une Structure de Don- 

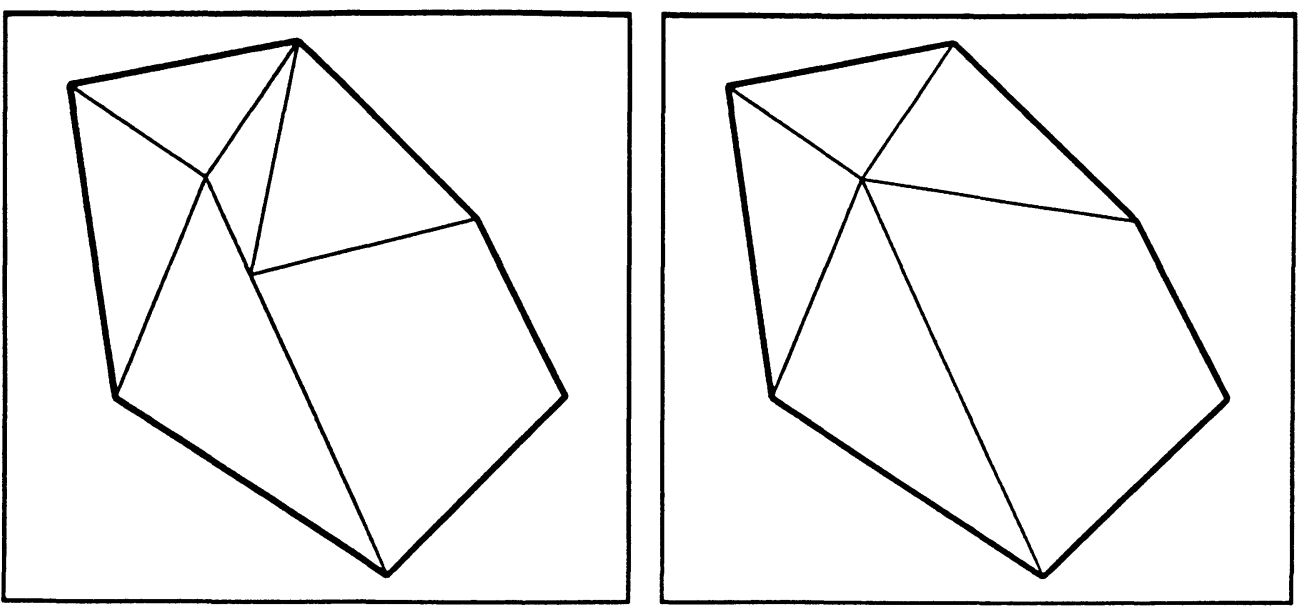

Fig. 1. - Mailles non conformes et conformes.

[Conformal and non conformal meshes.]

nées contenant sous forme condensée et accessible l'ensemble de ces valeurs.

- Point de vue géométrique : il concerne la description des éléments du maillage, ceux-ci peuvent être connus de différentes façons :

1. via la liste de leurs sommets et des définitions implicites de leurs arêtes et faces à partir de ces sommets ;

2. de manière descendante: les éléments sont décrits comme des volumes, ceux-ci sont connus via leurs faces, ces dernières le sont par leurs arêtes, elles-mêmes étant référencées par leurs deux sommets extrémités ;

3. d'autres formes de description sont envisageables, en particulier selon la nature des calculs que l'on désire réaliser.

- Point de vue physique : pour connaître la nature d'un élément (le matériau dont il est constitué), il est commode de lui associer un numéro de matériau; pour pouvoir définir les conditions limites du problème, il est naturel d'affecter un numéro de référence à chaque constituant des éléments (faces, arêtes et nœuds).

De la sorte une description par lots des valeurs physiques est obtenue; tous les éléments de numéro de matériau donné sont de tel ou tel type, toutes les faces de numéro de référence donné sont soumises à telle ou telle sollicitation.

La Structure de Données permettant de stocker l'ensemble de ces valeurs doit être conçue de façon à permettre un accès rapide à telle ou telle information. La solution à retenir, dépendant du type de problème traité, n'est pas unique ; par suite il n'est pas surprenant de constater qu'il n'existe pas une structuration unique [24] pour le stockage des maillages.

\section{Méthodologie.}

Pour créer un maillage, l'approche la plus naturelle se base sur la conception descendante et sur la réalisation ascendante, voir par exemple [13]. Cette façon de procéder (voir Fig. 2) présente de nombreux avantages : un domaine complexe est décomposé en sous-parties plus simples et bien adaptées aux algorithmes de maillages, chacune de ces parties est décrite de nouveau de façon descendante (volumes, faces, lignes puis points). Ensuite, la réalisation part des définitions les plus basses pour remonter aux items de rang plus élevé (sans perte d'informations) : le maillage des parties simples est d'abord construit puis, pas à pas, l'objet final est défini.

Cette méthode assure de manière évidente une garantie sur le résultat final au sens où si les maillages «simples » sont corrects, le maillage final l'est aussi.

\section{Algorithmes de génération.}

Dans cette section nous allons présenter les notions essentielles ayant trait aux principales méthodes actuellement utilisées ou faisant l'objet de recherches dans le domaine de la génération des maillages.

\subsection{GÉNÉRATEURS DE MAILLAGES EN DIMENSION} 2. - Cinq classes de générateurs sont couramment utilisées pour la création de maillages en dimension 2. Les maillages ainsi produits pourront être par la suite manipulés et recombinés de façon à créer de nouveaux maillages de complexité plus grande.

5.1.1. Définition manuelle. - A partir de la donnée de toutes les informations constitutives d'un maillage, on construit la Structure de Données associée à celui-ci. Cette approche convient dans le cas où peu d'éléments sont à créer (il faudra en effet les décrire 


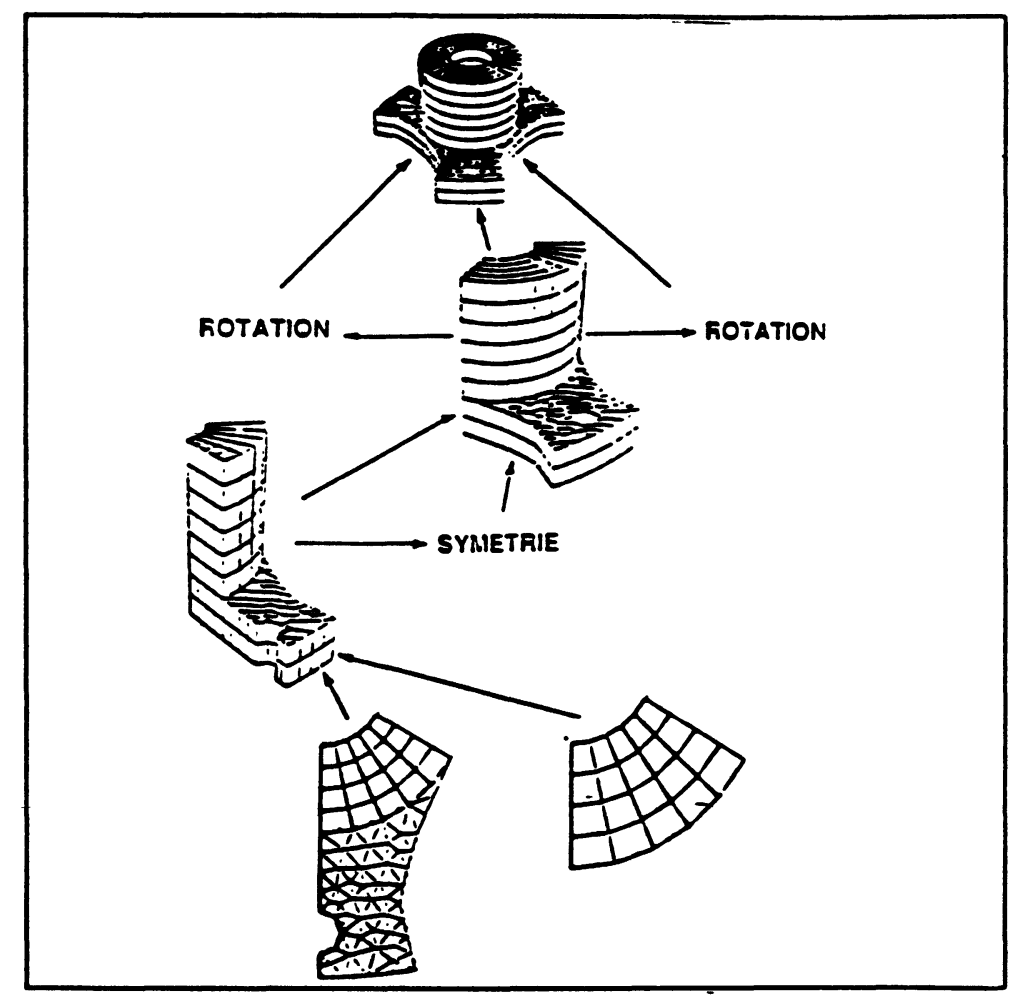

Fig. 2. - Conception descendante et construction ascendante.

[Top-down conception and bottom-up construction.]

un à un) ou dans celui où il est possible de définir (par exemple à l'aide d'un programme) tant les coordonnées des sommets du maillage que les liaisons entre ces sommets. Le maillage résultat pourra par la suite être, par exemple, redécoupé afin d'obtenir un recouvrement plus fin et plus fidèle du domaine.

5.1.2. Méthode de "transport-projection ». - Par cette approche [11] on pourra créer le maillage de tout domaine de topologie simple (un segment, un triangle ou un quadrangle déformé). La partition du domaine sera liée à la position et aux nombres de points présents sur son contour. La donnée de ce mailleur est donc une discrétisation du contour du domaine traité, c'est-à-dire un maillage de ce points sur deux côtés logiquement connectés est supposé identique.

Cette technique se décompose en plusieurs phases :

1. transport sur les côtés de l'élément unité de même nature que le domaine des points du contour de ce dernier en conservant les distances relatives entre ces points ;

2. maillage canonique de l'élément unité. Ce maillage revient à connecter les points se correspondant d'un côté à l'autre du domaine unité ;
3. transport de ce maillagè de référence sur le domaine réel. Soit $\hat{\mathbf{M}}$ un point du maillage canonique, de coordonnées $\hat{x}$ et $\hat{y}$, pour un domaine topologiquement analogue à un triangle on utilise la transformation suivante :

$$
\begin{aligned}
\mathrm{M}= & \frac{1-\hat{x}-\hat{y}}{1-\hat{x}} f_{1}(\hat{x})+\frac{\hat{x}}{1-\hat{y}} f_{2}(\hat{y}) \\
& +\frac{\hat{y}}{\hat{x}+\hat{y}} F_{3}(1-\hat{x}-\hat{y})-\left(\frac{\hat{y}}{\hat{x}+\hat{y}}(1-\hat{x}-\hat{y}) a_{1}\right. \\
& \left.+\frac{1-\hat{x}-\hat{y}}{1-\hat{x}} \hat{x} a_{2}+\frac{\hat{x}}{1-\hat{y}} \hat{y} a_{3}\right)
\end{aligned}
$$

qui permet de connaître l'image $M$ du point

Tandis que pour un domaine topologiquement analogue à un quadrangle (voir Fig. 3) on utilise la transformation :

$$
\begin{aligned}
\mathbf{M}= & (1-\hat{y}) f_{1}(\hat{x})+\hat{x} f_{2}(\hat{y})+\hat{y} f_{3}(\hat{x}) \\
& +(1-\hat{x}) f_{4}(\hat{y})-\left((1-\hat{x})(1-\hat{y}) a_{1}\right. \\
& \left.+\hat{x}(1-\hat{y}) a_{2}+\hat{x} \hat{y} a_{3}+(1-\hat{x}) \hat{y} a_{4}\right)
\end{aligned}
$$

où les $f_{i}$ sont des paramétrisations des côtés du domaine et les $a_{i}$ ses sommets.

Ces transformations respectent les sommets et les 


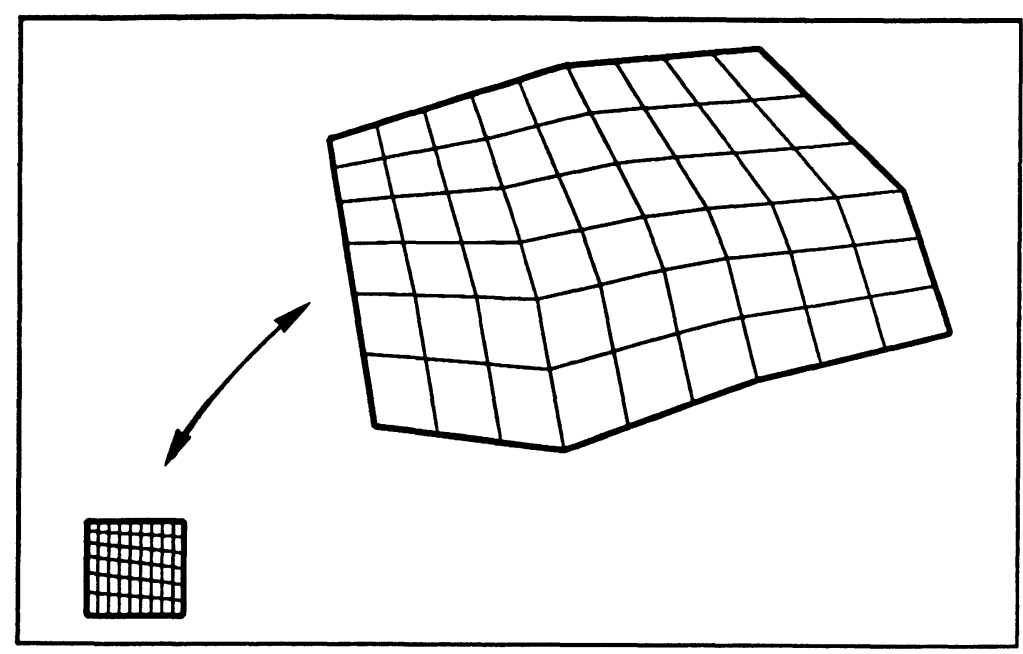

Fig. 3. - Cas d'un domaine de topologie quadrangulaire.

[Case of a domain with quadrangular topology.]

côtés du domaine (d'autres transformations assurant la même propriété sont envisageables).

Le maillage résultat sera composé d'éléments de même nature que le domaine traité considéré du point de vue topologique.

5.1.3. Méthode frontale. - Adaptée aux géométries a priori quelconques, ce type de mailleur va construire le recouvrement du domaine en triangles, à partir des points de son contour. Il utilise donc comme donnée ce contour et plus précisément une approximation polygonale de ce dernier sous la forme d'une liste de segments.

Le processus est itératif : à partir d'un contour donné, une analyse des angles formés par deux segments consécutifs et de leurs longueurs respectives permet de sélectionner une zone de départ.
Selon la taille des angles de cette zone un ou deux segments consécutifs sont considérés.

Soit $\alpha$ l'angle formé par 2 segments consécutifs du contour, trois situations sont prises en compte (voir Fig. 4) :

- $\alpha<\frac{\pi}{2}$, les 2 segments d'angle $\alpha$ sont retenus et vont être les 2 côtés du seul triangle créé.

- $\frac{\pi}{2} \leq \alpha \leq \frac{2 \pi}{3}$, à partir des 2 segments d'angle $\alpha$, on va générer un point interne et deux triangles. - $\frac{2 \pi}{3}<\alpha$, un seul segment est retenu, un triangle est construit avec ce segment comme côté et un point interne.

Un nouveau contour est formé en éliminant du contour actuel les arêtes du ou des éléments cons-

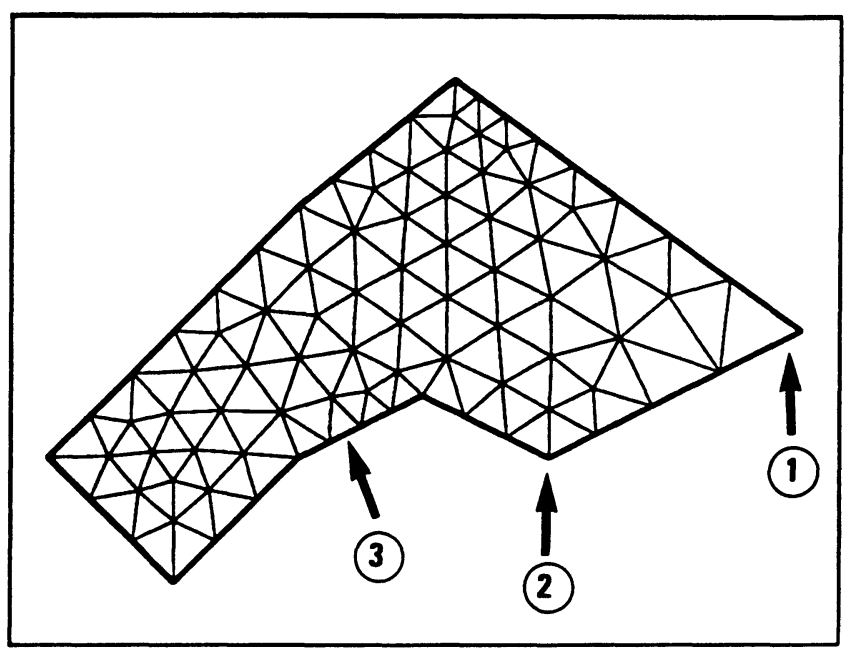

Fig. 4. - Constructions selon les situations.

[Constructions w.r.t. situations.] 
truits communes avec le contour initial et en insérant à leur place la ou les arêtes construites non communes à deux triangles. Ce nouveau contour est alors traité par la même méthode.

Quand le contour est réduit à l'ensemble vide, le maillage final est obtenu; il peut être régularisé pour obtenir des triangles de meilleure qualité.

5.1.4 Méthode de type Voronoï. - Pour des géométries absolument quelconques, cette méthode [30, $22,7]$ permet de construire un recouvrement en triangles, à partir, encore une fois, d'une partition polygonale du contour du domaine.

La méthode comprend plusieurs phases.

1. Création du nuage de points associé aux données, c'est-à-dire les points du contour du domaine.

2. Calcul de la position de 4 points supplémentaires tels que le quadrilatère formé par ceux-ci englobe tous les points du nuage.

3. Maillage de ce quadrilatère à l'aide de 2 triangles (soit $\mathrm{T}_{4}$ ce maillage).

4. Insertion, un à un, des points du nuage pour construire un maillage comprenant comme sommets d'éléments ces points.
Pour réaliser cette étape nous allons définir un processus de remaillage local permettant de créer un nouveau maillage à partir d'un maillage donné et d'un point interne à ce dernier de telle sorte que ce point soit un sommet d'éléments.

Soient donc :

- $\mathrm{T}_{n+4}$ une triangulation comprenant comme sommets les $n$ premiers points d'un nuage $\left(\mathrm{T}_{4}\right.$ est le recouvrement initial du quadrilatère englobant),

- $\mathrm{P}_{n+1}$ le point suivant de ce nuage.

D'après la phase 3 du schéma ci-dessus, le point $P_{n+1}$ est intérieur à $T_{n+4}$, plus précisément deux situations seulement sont possibles :

(a) $P_{n+1}$ est interne à un élément $E_{i}$ de $T_{n+4}$,

(b) $\mathrm{P}_{n+1}$ est sur l'arête commune à deux éléments $\mathrm{E}_{j}$ et $\mathrm{E}_{k}$ de $\mathrm{T}_{n+4}$.

Le troisième cas envisageable serait celui où $P_{n+1}$ est confondu avec l'un des points déjà présents dans le maillage, ce cas est impossible car les points du nuage donné sont supposés distincts.

$A$ partir de $E_{i}$ (ou des éléments $E_{j}$ et $E_{k}$ ), on construit par voisinage l'ensemble $\mathrm{D}$ des éléments de $\mathrm{T}_{n+4}$ tels que :

- $\mathrm{P}_{n+1}$ est interne au cercle circonscrit aux éléments de D.
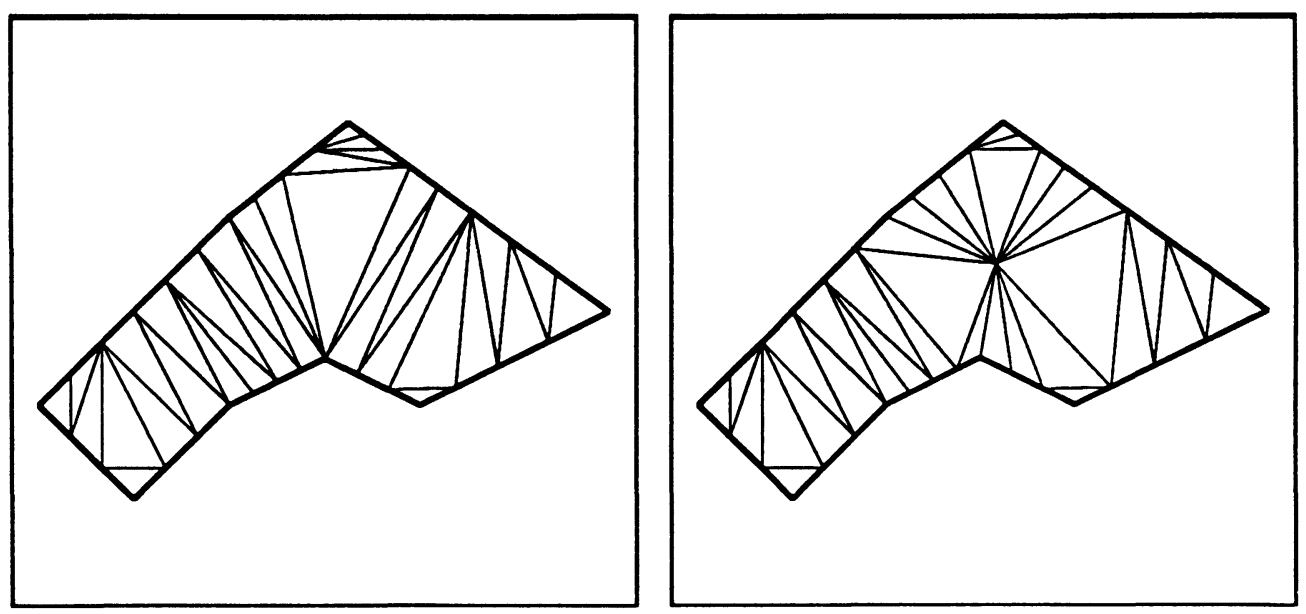

Fig. 5. - Insertion d'un point.

[Inserting a point.]

Nous renvoyons, par exemple, à [22] qui démontre que cet ensemble est étoilé par rapport au point $\mathrm{P}_{n+1}$. Par suite la construction de la triangulation $\mathrm{T}_{n+5}$ est menée de la façon suivante (voir Fig. 5) :

(a) mettre dans $T_{n+5}$ les éléments de $T_{n+4}$ non inclus dans $\mathrm{D}$,

(b) détruire les éléments de $\mathrm{D}$ et remailler cet ensemble en joignant le point $\mathbf{P}_{n+1}$ aux arêtes externes de $\mathrm{D}$.
Quand tous les points du nuage de départ ont été introduits selon cette méthode, le recouvrement en triangles $\mathrm{du}$ quadrilatère initial est obtenu. Ce maillage « $\mathrm{M} 1$ » comprend comme points tous les points donnés plus les 4 points supplémentaires créés lors de la phase 2 du schéma.

5. Reconnaissance des arêtes données dans ce maillage. Sous certaines hypothèses raisonnables [18] le maillage « M1 » contient dans la liste de ses 
arêtes toutes les arêtes du contour servant de donnée. Il est alors possible de marquer les triangles par rapport à leur position vis-à-vis des arêtes du contour.

6. Elimination des éléments extérieurs à l'objet traité. Les triangles étant marqués, il suffit de conserver seulement ceux qui sont internes au domaine pour obtenir un maillage de celui-ci, soit « $\mathrm{M} 2$ » ce maillage.

7. Création de points internes dans ce dernier maillage. Le maillage "M2» comprend comme sommets d'éléments uniquement les points du contour, par suite il est généralement impropre aux calculs. En utilisant le processus d'insertion de points dans un maillage existant, mentionné ci-dessus, on va créer des points internes par barycentrage pondéré de façon à obtenir un maillage « M3 » constitué de triangles de bonne qualité.
8. Régularisation éventuelle du résultat. Le maillage peut être maintenant régularisé par déplacement des points internes au barycentre de leurs voisins.

5.1.5 Méthode basée sur la notion de quadtree. - Ce type de mailleur $[5,31]$ va construire le recouvrement du domaine considéré à partir des points de son contour.

1. Un premier rectangle est formé qui contient tous les points du contour. La méthode consiste à découper, à partir de ce rectangle initial, chaque rectangle (ou père) en 4 rectangles (ou fils), de façon récursive, jusqu'à obtention d'une partition du rectangle initial en éléments quadrangulaires tels que chacun contienne au plus un seul point du contour. Cette dernière propriété permet de contrôler la taille de chaque maille (voir Fig. 6).
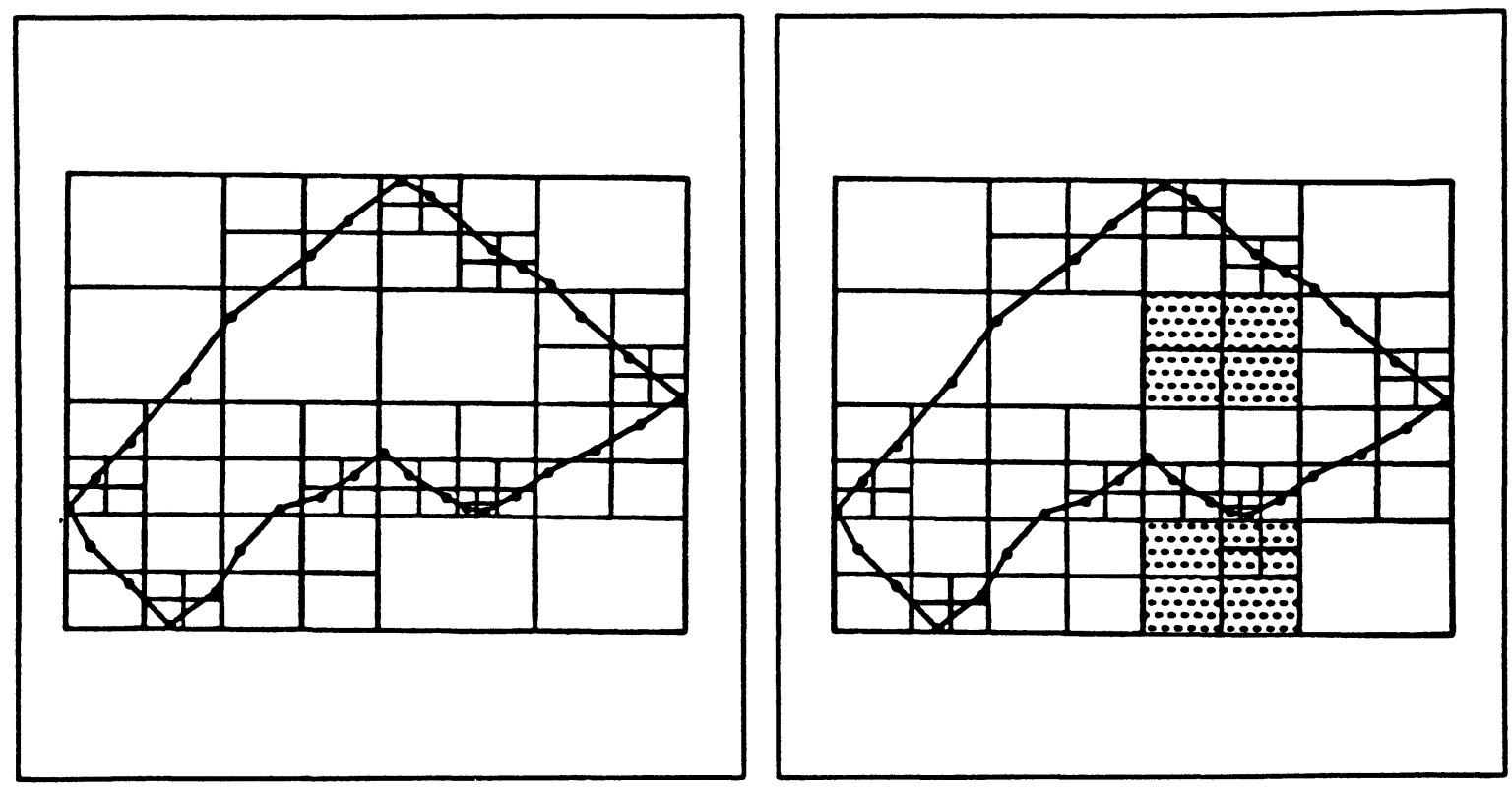

Fig. 6. - Structure initiale et équilibrage.

[Initial structure and balanced one.]

2. La partition issue de l'étape ci-dessus est équilibrée (voir Fig. 6) par définition de fils de telle sorte qu'au plus un point intermédiaire existe sur une arête d'une maille.

3. Une analyse des mailles ainsi générées est menée :

- maille extérieure ne contenant aucun point: une telle maille est détruite,

- maille intérieure ne contenant aucun point : une telle maille produit un quadrangle (qui peut être découpé en triangles) si ses arêtes ne possèdent pas de points intermédiaires ou est découpé en triangles dans le cas inverse,
- maille contenant un «morceau» du contour : les points intersections du contour et des arêtes de la maille sont créés, on définit de la sorte une partition de la maille dont on ne garde que la partie interne au domaine (le contour final du maillage est donc formé à ce stade).

4. Une régularisation des points internes est alors effectuée (les points internes sont les sommets hors contour des mailles de la partition issue de l'étape 2 de ce processus).

Remarque: Il existe de nombreuses variantes de cette méthode. 
5.2 GÉNÉRATEURS DE MAILlageS EN DIMENSION 3. - Huit classes de générateurs sont actuellement envisageables pour la création de maillages en dimension 3. Comme en dimension 2, les maillages ainsi produits pourront être par la suite manipulés et recombinés de façon à créer de nouveaux maillages plus complexes.

5.2.1 Définition manuelle. - A partir de la donnée de toutes les informations constitutives d'un mail- lage, la Structure de Données associée à celui-ci est construite. Les cas d'application de cette approche restent ceux mentionnés dans le cadre bidimensionnel vu ci-dessus.

5.2.2 Méthode de type différences finies. - Adaptée aux géométries de type cubique, cette méthode va construire le recouvrement du domaine en hexaèdres (qui pourront être redécoupés par la suite) à partir de la donnée de points selon 3 directions.
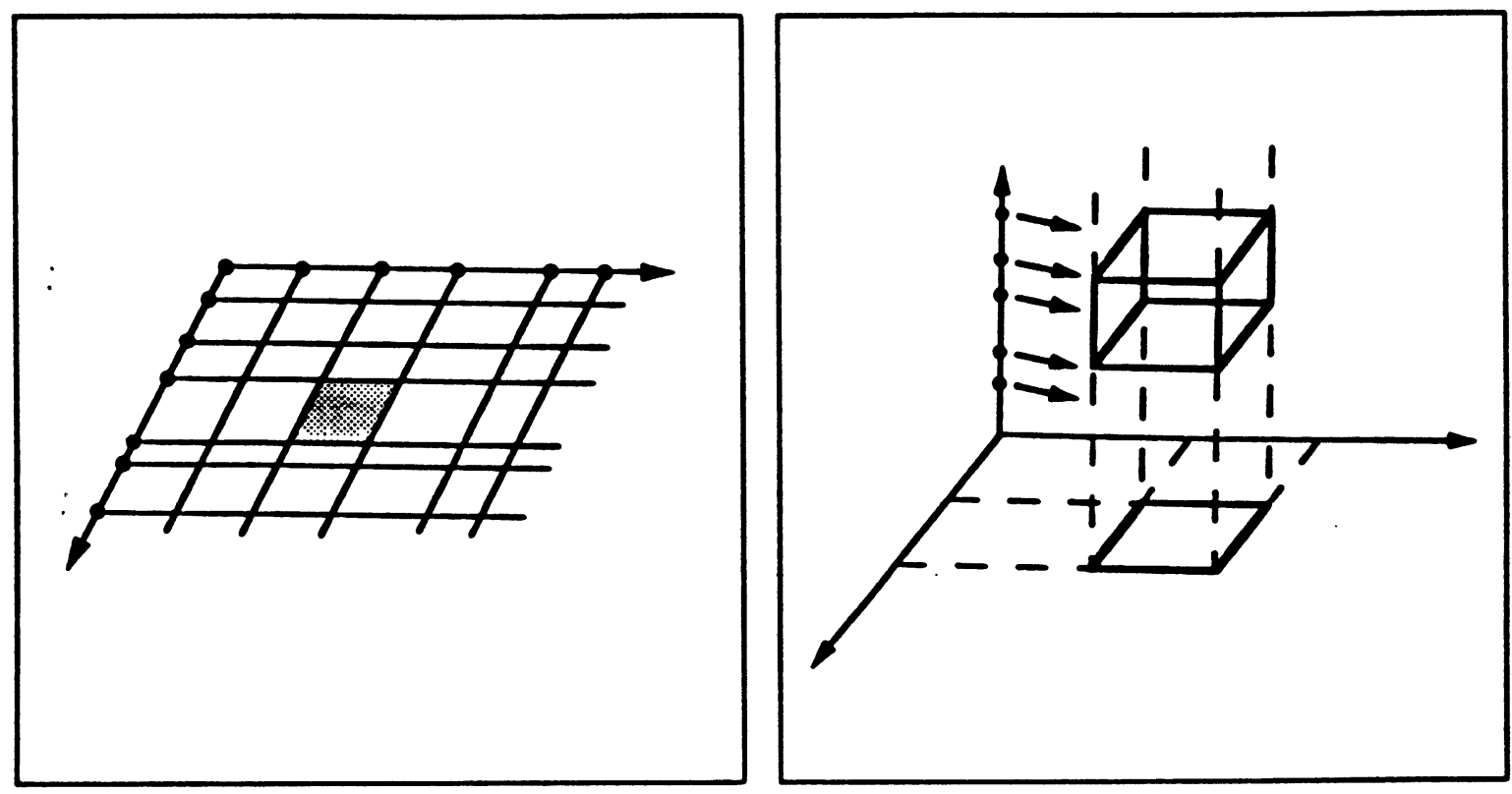

Fig. 7. - Partition 2D et construction 3D associée.

[2D partition and associated $3 \mathrm{D}$ construction.]

En se plaçant dans le plan défini par les deux premières directions un maillage est construit. Il est formé de quadrangles dont les sommets sont déduits par «convolution » des deux ensembles de points associés aux directions. Chaque élément de ce maillage sert alors à construire un empilement d'hexaèdres par " convolution " avec les points de la troisième direction (voir Fig. 7). Relativement simple dans son principe, cette méthode permet déjà de résoudre le problème du maillage de domaines assez complexes entrant dans le cadre de cette topologie particulière.

5.2.3 Méthode de "translation-empilement". - Par cette approche [14] le maillage de tout domaine de topologie cylindrique pourra être créé à partir de la connaissance d'un maillage $2 \mathrm{D}$ de référence dont seront déduites les différentes couches d'éléments 3D. La donnée de ce mailleur est donc le maillage 2D et la définition des sections le long de la génératrice du «cylindre ».

Cette méthode peut donc être vue comme une généralisation de la précédente.
A chaque segment du maillage $2 \mathrm{D}$ est associée une série de quadrangles dans l'espace, au triangle sont associés des pentaèdres, au quadrangle des hexaèdres.

Afin de permettre de traiter des domaines comprenant l'axe du cylindre associé, il est nécessaire de considérer les cas de dégénérescence possible (voir Fig. 8), un quadrangle donnant lieu à la création de pentaèdres et non plus d'hexaèdres.

\subsubsection{Méthode de blocs avec "transport-projec-}

approche [11] permet de construire le recouvrement en Eléments Finis à partir du découpage structuré d'un maillage grossier du domaine. Ce dernier recouvrement est composé de blocs de géométrie simple (lignes, triangles, quadrangles, tétraèdres, pentaèdres, hexaèdres). Le découpage est guidé par la présence de points sur les arêtes du maillage grossier et sera lié à leurs positions relatives.

Dans son principe cette méthode est identique à celle présentée dans le cadre 2D dans le cas d'un seul bloc. 

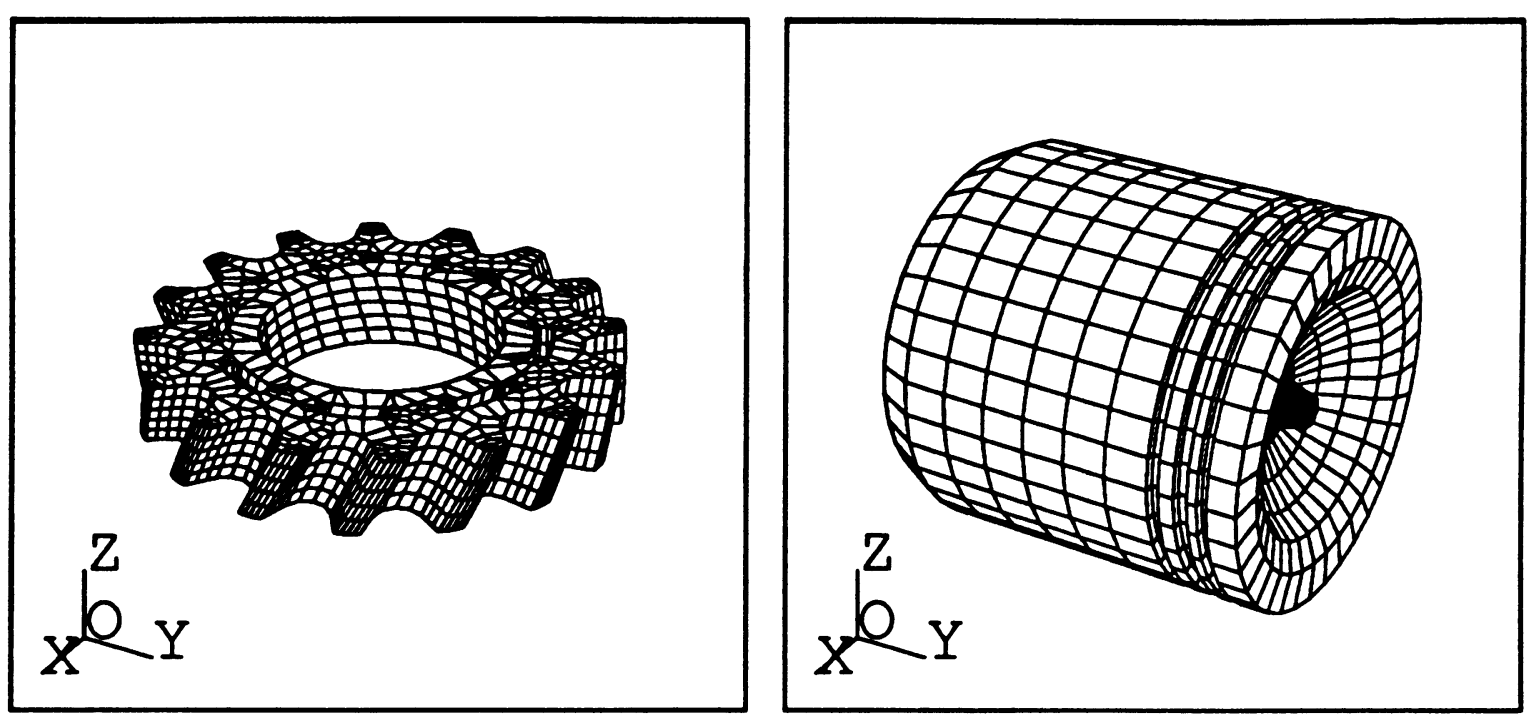

Fig. 8. - Cas standard et cas dégénéré.

[Standard case and degenerated one.]

On peut résumer cette approche de la façon suivante :

1. Maillage des faces des éléments grossiers. Les points des arêtes sont utilisés et par application du procédé vu dans le cadre $2 \mathrm{D}$, on génère la partition en sous-éléments de chaque face.

2. Maillage des blocs. Considérant chaque élément du maillage grossier et le maillage de leurs faces, on va créer le maillage du bloc par découpage de celui-ci par :

- transport sur les faces de l'élément unité de même nature que le bloc des points des faces de ce dernier en conservant les distances relatives entre ces points ;

- maillage canonique de l'élément unité. Ce maillage revient à connecter les points se correspondant d'une face à l'autre du domaine unité ;

- transport de ce maillage de référence sur le domaine réel. Soit $\hat{\mathbf{M}}$ un point du maillage canonique, de coordonnées $\hat{x}, \hat{y}$ et $\hat{z}$; dans le cas d'un bloc topologiquement analogue à un tétraèdre on utilise la transformation suivante :

$$
\hat{\mathbf{M}} \Rightarrow \tilde{\mathbf{M}}=F(\hat{x}, \hat{y}, \hat{z})
$$

avec :

$$
F(x, y, z)=\sum_{i=1}^{4} P_{i}(x, y, z)
$$

où on $\mathrm{a}$ :

$$
\begin{gathered}
P_{1}(x, y, z)=1-x-y-z \\
P_{2}(x, y, z)=x \\
P_{3}(x, y, z)=y \\
P_{4}(x, y, z)=z
\end{gathered}
$$

qui permet de connaître l'image $\tilde{M}$ du point M.

A partir de ce point $\tilde{\mathbf{M}}$ on utilise une méthode de déformation de mailles pour créer le point $M$ correspondant (voir Fig. 9) :

$$
\tilde{\mathrm{M}} \Rightarrow \mathrm{M}=D(\tilde{\mathrm{M}}, \text { frontière }) \text {. }
$$

La fonction $D$ prend en compte l'ensemble des points des faces du bloc réel et repositionne le point traité comme barycentre pondéré de ceux-ci. Cette technique permet de mieux traiter les blocs non convexes (la méthode ne garantissant pas que $\tilde{\mathbf{M}}$ soit interne au bloc dès lors que $\mathrm{M}$ est interne au bloc unité).

3. Enumération des éléments issus du découpage. Pour obtenir les éléments composant le maillage du bloc, il suffit d'énumérer leurs sommets.

4. Recollement les maillages des différents blocs. Cette opération est triviale dès lors que les points des faces communes à deux blocs sont numérotés de façon globale.

5.2.5 Méthode frontale. - Adapté aux géométries a priori quelconques, ce type de mailleur $[23,20]$ va construire le recouvrement du domaine en tétraèdres, à partir des faces de son contour. Il utilise donc comme donnée ce contour et plus précisément une approximation polyédrique de ce dernier sous la forme d'une liste de triangles.

Le processus est itératif : à partir d'un contour donné, une analyse des propriétés (en terme de taille et d'angles) des faces du contour et de leur voisinage permet de sélectionner une zone de départ.

Selon les cas un élément est créé avec les faces retenues où un point interne est généré qui sert de 

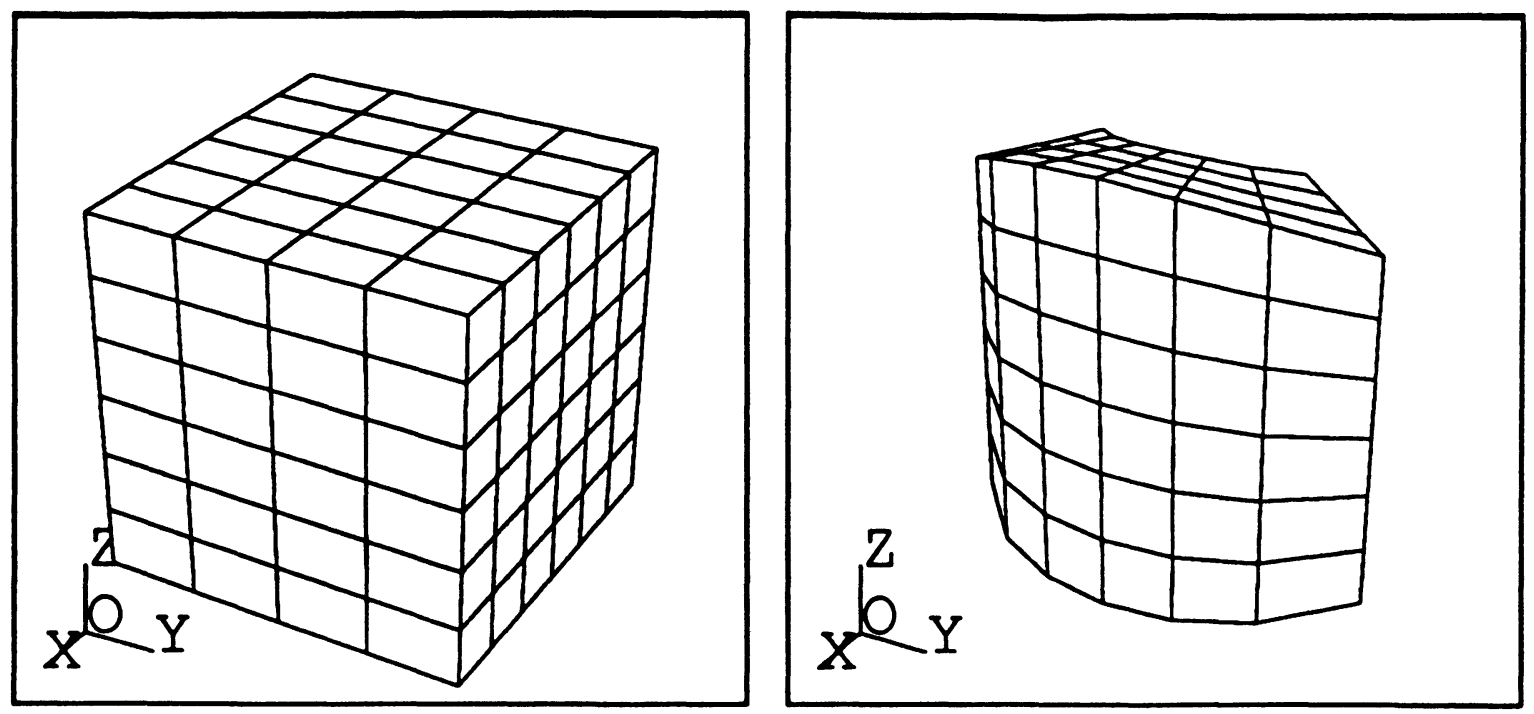

Fig. 9. - Transport de la partition canonique sur l'objet réel.

[Mapping of the canonical partition on the real geometry.]

sommet aux éléments le joignant aux faces sélectionnées.

Un nouveau contour est formé en éliminant du contour actuel les faces du ou des éléments construits situées sur le contour initial et en insérant à leur place la ou les faces construites non communes à deux tétraèdres. Ce nouveau contour est alors traité par la même méthode.

Quand le contour est vide le maillage final est obtenu, il peut être régularisé pour obtenir des tétraèdres de meilleure qualité.

5.2.6 Méthode de type Voronoï. - Pour des géométries absolument quelconques, cette méthode [30, $25,17,18,1,3,4]$ permet de construire un recouvrement en tétraèdres, à partir des faces décrivant le contour du domaine. La méthode (comme en dimension 2) comprend plusieurs phases :

1. Création du nuage de points associé aux données, c'est-à-dire les points des faces du contour du domaine.

2. Calcul de la position de 8 points supplémentaires tels que l'hexaèdre formé par ceux-ci englobe

3. Maillage de cette boîte à l'aide de 5 tétraèdres (soit $\mathrm{T}_{8}$ ce maillage).

4. Insertion, un à un, des points du nuage pour construire un maillage comprenant comme sommets d'éléments ces points.

Pour réaliser cette étape nous allons définir un processus de remaillage local permettant de créer un nouveau maillage à partir d'un maillage donné et d'un point interne à ce dernier de telle sorte que ce point soit un sommet d'éléments.
Soient donc :

- $\mathrm{T}_{n+8}$ une triangulation comprenant comme sommets les $n$ premiers points d'un nuage ( $\mathrm{T}_{8}$ est le recouvrement initial de la boîte englobante),

- $\mathbf{P}_{n+1}$ le point suivant de ce nuage.

D'après la phase $3 \mathrm{du}$ schéma ci-dessus, le point $\mathbf{P}_{n+1}$ est intérieur à $\mathrm{T}_{n+8}$, plus précisément trois situations seulement sont présentes :

(a) $P_{n+1}$ est interne à un élément $E_{i}$ de $T_{n+8}$,

(b) $\mathrm{P}_{n+1}$ est sur la face commune à deux éléments $\mathrm{E}_{j}$ et $\mathrm{E}_{k}$ de $\mathrm{T}_{n+8}$,

(c) $\mathrm{P}_{n+1}$ est sur l'arête commune à plusieurs éléments $\mathrm{E}_{k}$ de $\mathrm{T}_{n+8}$.

Le quatrième cas envisageable serait celui où $\mathbf{P}_{n+1}$ est confondu avec l'un des points déjà présents dans le maillage, ce cas est impossible (voir le cadre de la dimension 2).

A partir de $\mathrm{E}_{i}$ (ou de l'ensemble des éléments sélectionnés) et par voisinage un ensemble D d'éléments de $T_{n+8}$ est construit, il est tel que :

- $\mathrm{P}_{n+1}$ est interne au boule circonscrite aux éléments de $\mathrm{D}$.

n emontre que cet ensem e es e oi é par rapport au point $\mathbf{P}_{n+1}$. Par suite on peut construire la triangulation $T_{n+9}$ de la façon suivante :

(a) mettre dans $T_{n+9}$ les éléments de $T_{n+8}$ non inclus dans $\mathrm{D}$,

(b) détruire les éléments de $\mathrm{D}$ et remailler cet ensemble en joignant le point $\mathbf{P}_{n+1}$ aux faces externes de $\mathrm{D}$.

Quand tous les points du nuage de départ ont été introduits selon cette méthode, le recouvrement en tétraèdres de la boîte initiale est obtenu. Ce maillage 
« $\mathrm{M} 1$ » comprend comme points tous les points donnés plus les 8 points supplémentaires créés lors de la phase 2 du schéma ci-dessus.

5. Reconnaissance des faces données dans ce maillage. On cherche si le maillage " $\mathrm{M} 1$ » contient dans la liste de ses faces toutes les faces du contour servant de donnée; en dimension 3 ce résultat n'est pas acquis (on peut construire très simplement des exemples en ce sens [16]), par suite il est nécessaire d'utiliser une méthode de modification locale (voir Fig. 10) des éléments permettant de recréer toutes les faces données. Une fois cette opération effectuée il est possible de marquer les tétraèdres par rapport à leur position vis-à-vis des faces du contour.
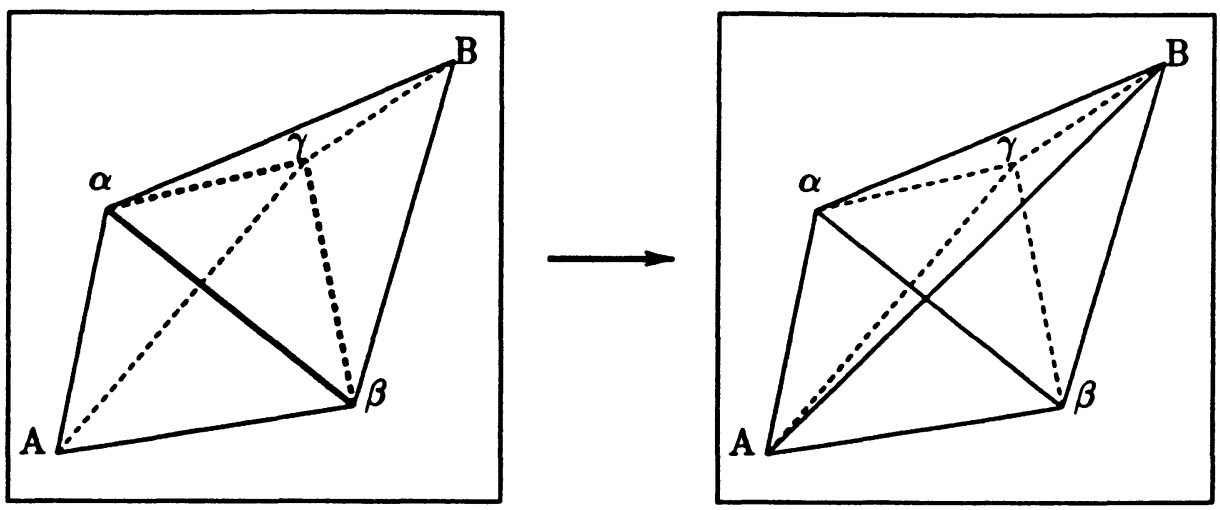

Fig. 10. - Modifications locales.

[Local modifications.]

6. Elimination des éléments extérieurs à l'objet traité. Les tétraèdres étant marqués, il suffit de conserver seulement ceux qui sont internes au domaine pour obtenir un maillage de celui-ci, soit «M2 » ce maillage.

7. Création de points internes dans ce dernier maillage. Le maillage «M2 » comprend comme sommets d'éléments uniquement les points du contour, par suite il est généralement impropre aux calculs. En modifiant localement « M2 » et en utilisant le processus d'insertion de points vu ci-dessus, certains éléments vont être réécrits et des points internes vont être créés par barycentrage pondéré de façon à obtenir un maillage « M3 » constitué de tétraèdres de bonne qualité.

8. Régularisation éventuelle du résultat. Le maillage peut être maintenant régularisé par déplacement des points internes au barycentre de leurs voisins.

5.2.7 Méthode basée sur la notion d'octree. - La méthode présentée dans le cadre de la dimension 2 peut se généraliser à la dimension $3[31,26]$ la notion de quadtree est alors remplacée par celle d'octree : un père est un hexaèdre qui permet de définir 0 ou 8 fils. Le découpage de la partition composée d'hexaèdres est alors une opération plus complexe qu'en dimension 2, en particulier pour obtenir un maillage conforme, les éléments sont découpés en tétraèdres.

\subsubsection{Mailleur de surfaces dans l'espace.}

- A partir d'un maillage 2D et d'une fonction de projection dans l'espace, on génère le maillage surfacique correspondant.

- D'une définition paramétrée ou sous forme de carreaux de la surface, on déduit le maillage de celleci. Les principales méthodes utilisées sont les techniques de "projection » pour des surfaces décrites par blocs de topologie simple, ou de type «Voronoï» pour des contours quelconques; il s'agit des approches vues dans le cadre 2D généralisées à l'espace de la surface.

\subsubsection{Cas particuliers.}

- A côté de ces générateurs généraux, il convient de mentionner d'autres outils de création de maillages adaptés à certains cas particuliers (Treillis, poutres, raccords, charnières ...).

- Dans le cadre des maillages structurés l'emploi de techniques algébriques permet de ramener le problème sur un domaine de calcul de type rectangle (en dimension 2) par un changement de variables. Nous renvoyons à [29] pour ce type de méthodes.

- Pour des simulations autres que par les Eléments Finis (Volumes Finies, Différences Finis, méthodes particulaires, ...) la notion même de maillage est à redéfinir.

5.3 Programmes de MANIPULATION DE MAILlAGES. - Plusieurs types de manipulations sont possibles : les transformations géométriques usuelles, des transformations topologiques, le recollement de 
maillages, les modifications propres aux Eléments Finis et enfin la visualisation sous différentes formes.

5.3.1 Modifications géométriques. - Elles permettent d'obtenir le maillage déduit par translation, symétrie, rotation ou dilatation anisotrope d'un maillage initial.

\subsubsection{Découpage local ou global.}

1. Découpage local. Plusieurs algorithmes sont envisageables pour affiner localement un maillage au voisinage de certains de ses sommets. Un tel processus peut être basé sur :
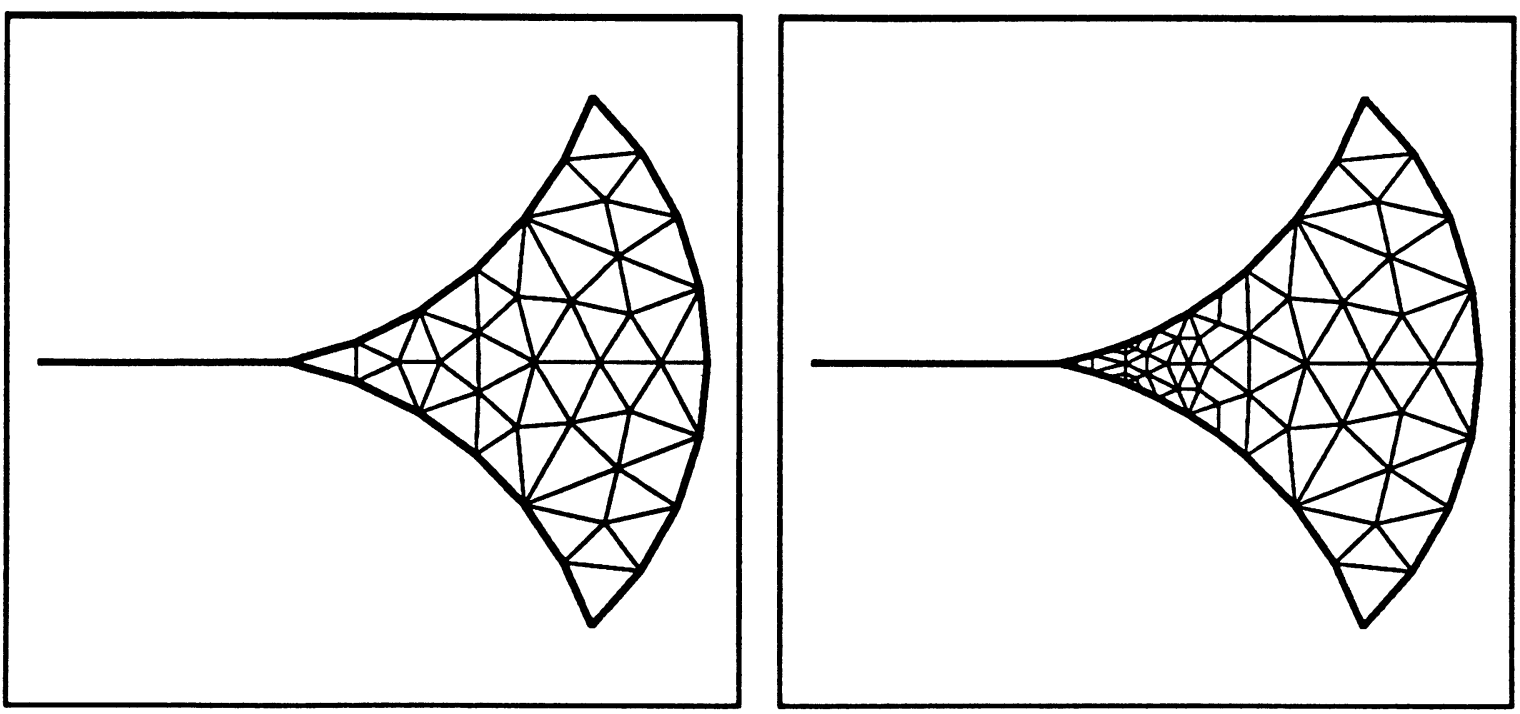

Fig. 11. - Affinage local.

[Local refinement.]

2. Découpage global. Un maillage et $N$ un paramètre de découpe sont donnés. Sur chaque arête du maillage sont définis $N$ points intermédiaires, le procédé de partition canonique, déjà décrit, permet alors de découper tous les éléments en $(N+1)$ éléments (cas du segment), $(N+1)^{2}$ éléments (cas du triangle ou du quadrangle) ou en $(N+1)^{3}$ éléments (pour les tétraèdres, pentaèdres et hexaèu meme ype que e emen e epar .

3. Changement de type. Cette opération appliquée aux quadrangles permet de créer deux ou quatre triangles, appliquée aux pentaèdres elle donne 3 tétraèdres tandis que pour les hexaèdres elle fournit 5 ou 6 tétraèdres.

5.3.3 Recollement de 2 maillages. - Le recollement consiste à construire le maillage résultant de la juxtaposition de deux maillages initiaux par identification des zones de contact communes (points, arêtes et faces). Afin d'effectuer cette opération très
- Un remaillage local autour de points sélectionnés. Dans le cas de maillages composés de simplexes (triangles en dimension 2 ou tétraèdres en dimension 3) une méthode du type de celle présentée dans le cas des générateurs "Voronoï » est possible ou des modifications locales peuvent être effectuées [27].

- Une découpe des éléments sélectionnés par bissection, trisection, ... peut être appliquée (voir Fig. 11) qui engendre par voisinage et pour des raisons de conformité une découpe des éléments voisins. Facile à mettre en œuvre en dimension 2 , ce type de modifications locales est plus délicat à concevoir en dimension 3. rapidement une technique de h-coding peut être envisagée; elle permet de retrouver les items communs aux deux maillages.

5.3.4 Régularisation. - A côté des techniques de barycentrage pondéré permettant de régulariser un maillage figurent des méthodes itératives qui sont basées sur un déplacement des points internes à un obtenu (en terme de qualité).

\subsubsection{Manipulations diverses.}

1. Définition des nœuds. Pour utiliser dans le calcul des Eléments Finis différents des éléments P1 (dont les seuls nœuds sont les sommets) il convient de rajouter les nœuds qui ne sont pas des sommets (sur les arêtes, les faces ou à l'intérieur) et, éventuellement, d'indiquer que les sommets ne sont pas des nœuds (voir Fig. 12).

2. Renumérotation. Afin de minimiser la place 

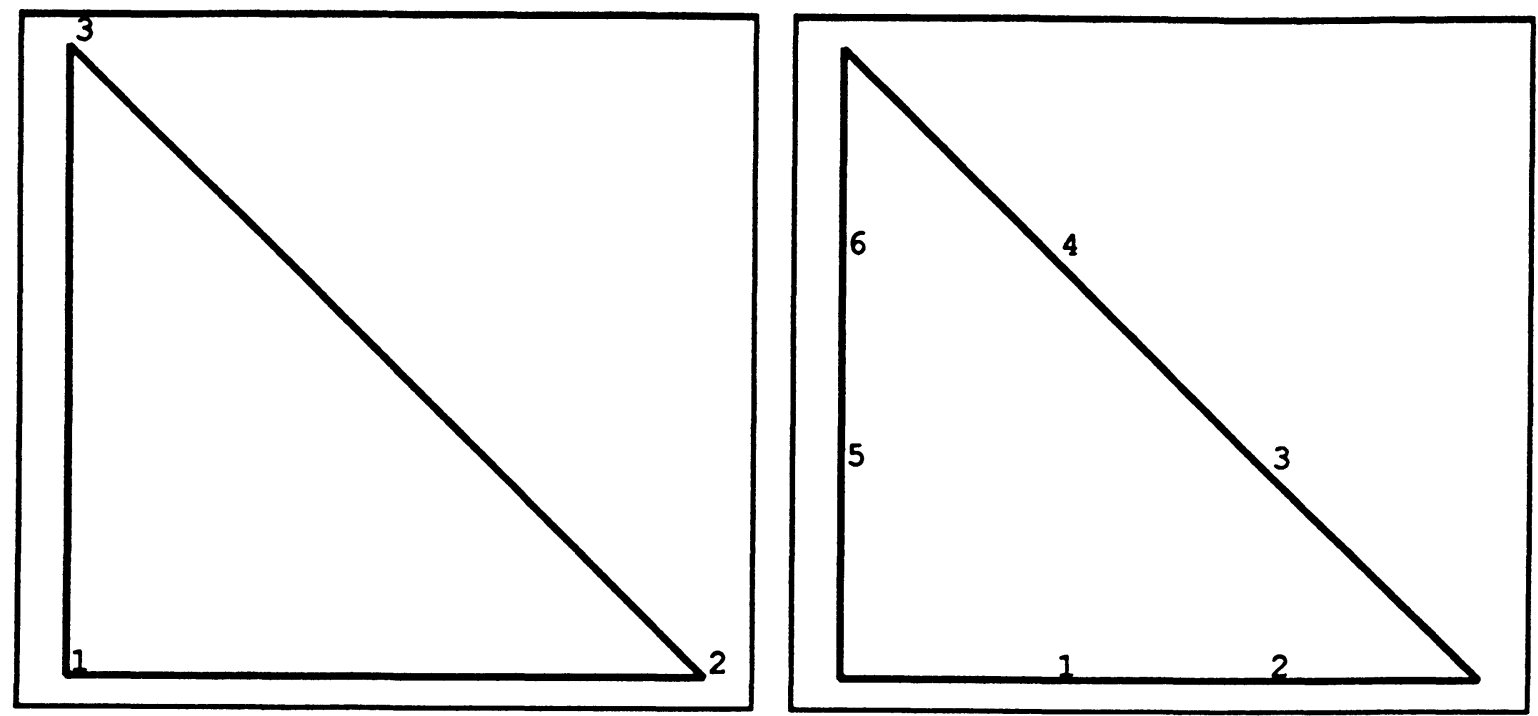

Fig. 12. - Définition des nœuds.

[Definition of nodes.]

requise pour stocker les matrices calculées lors du traitement numérique, il est souvent utile de renuméroter les nœuds et éventuellement les éléments du maillage.

3. ...

5.3.6 Adaptation. - Un couplage des techniques de régularisation et de découpe locale des mailles permet d'envisager la construction de maillages adaptés à la physique des problèmes traités. Dans une première phase un maillage du domaine est construit par l'emploi des approches décrites dans ce papier puis un premier calcul de la solution du problème est effectué. Après choix d'un critère pertinent (gradient de la solution ou d'un champ dérivé de celle-ci), on détecte dans le maillage initial les zones à adapter (par raffinement ou par déraffinement) et on génère un nouveau maillage mieux adapté au problème ; ce processus est généralement itératif.

5.3.7 Visualisations et appréciations des maillages. - Pour apprécier les maillages construits, l'utilisation de moyens graphiques évolués (surtout dans le cadre de la dimension 3 ) est nécessaire; de plus un certain nombre de contrôles peut être effectué de manière purement «calculatoire ".

1. Visualisations. Par mouvements de l'observateur, définitions de zooms, possibilités de "shrink " (rétrécissement des faces), utilisation de la couleur, prise en compte de la notion de faces cachées, ... les postprocesseurs graphiques (voir par exemple [15, 19]) permettent de juger les maillages.

De plus pour apprécier et communiquer les résultats des calculs (champs de vitesses, de pressions, de températures, ...) ces outils doivent en particulier permettre de représenter de manière efficace les grandeurs associées (scalaires, vecteurs, tenseurs ...).

2. Appréciation. Quand la simple observation se révèle fastidieuse ou impossible, on doit utiliser une approche automatique : trouver un critère mesurant la qualité du maillage ou de la solution calculée, calculer la valeur de ce critère puis représenter sous une forme adaptée cette mesure.

5.4 LES DONNÉES A FOURNIR. - Les données sont, du point de vue formel et en accord avec la méthodologie proposée ci-dessus, définies de manière arborescente. Plus précisément elles sont organisées selon une hiérarchie croissante : les items simples servant à définir les items plus complexes.

5.4.1 Nature des données. - Selon la méthode utilisée, les données consistent en tout ou partie de :

1. Une liste de Points caractéristiques. Ils sont connus via leurs 2 (ou 3) coordonnées et leur numéro de référence.

2. Une liste de Lignes caractéristiques. Elles sont construites à partir des points précédemment définis. Cette description concerne leur géométrie, précise leur numéro de référence et indique leur partition en sous-segments.

3. Une liste de Faces. Elles sont décrites via les items ci-dessus, un numéro de référence leur est associé.

4. Des Contours. Un contour (dimension 2) est l'union de lignes caractéristiques, il sert à définir un domaine à mailler, à celui-ci sera affecté un numéro de sous-domaine.

5. Des Volumes. Ils seront définis au travers des items de rang inférieur. 
6. Des Maillages préexistants. Ceux-ci serviront de données pour l'opération envisagée (constructions de maillages, transformations géométriques ou topologiques).

5.4.2 Construction des données. - Les données utiles pour créer un maillage peuvent rapidement atteindre une quantité très volumineuse. Pour faciliter les saisies correspondantes, plusieurs approches existent :

- création de fichier de commandes selon un formalisme défini. Dans ce fichier sont présentes les requêtes (sous forme de mots-clés ou de code) et les valeurs numériques associées (coordonnées, numéro de traitement, valeurs des coefficients physiques ...) ;

Ces fichiers sont à remplir à la main ou sont générés via des préprocesseurs "intelligents »;

- définition des valeurs liées à la géométrie via des systèmes de D.A.O. (Dessin assisté par ordinateur) ou de C.A.O. (Conception assistée par ordinateur);

- écriture de programmes contenant les données en langage de bas niveau (Fortran par exemple) ou en langage évolué (utilisation de grammaire, ...);

\section{- ...}

Notons l'apparition de la norme SET (Afnor) dont le but est de définir un standard permettant les descriptions liées aux Eléments Finis dans leurs aspects géométriques en particulier.

5.4.3 Quelques logiciels de maillage. - La plupart des codes d'Eléments Finis contiennent un ensemble de processeurs permettant la génération des maillages ; si tel n'est pas le cas soit ils ont prédéfini une structure de données correspondante que l'utilisateur doit créer selon ces spécifications, soit ils sont couplés à des logiciels de C.A.O.

Parmi les nombreux codes existants, nous pouvons mentionner :

- Ansys

- Aska

- Castem du CEA

- Catia de AMD BA [2]<smiles></smiles>

- Emc2 de l'INRIA [21]

- Euclid de Matra Datavision [28]

- Flux3d de l'INPG (Grenoble) [8]

- Mef/Mosaic de CSI/UTC (Compiègne)

- Modulef (l'auteur assure la responsabilité de la partie maillage de cette bibliothèque d'Eléments Finis) $[12,10,9]$.

- Nastran

- Patran

- Samcef du L.T.A.S. de Liège

- Simail de Simulog
- Supertab Geomod de S.D.R.C.

- Systus et Titus de Framatome

- ...

6. Quelques exemples de maillages en dimension 2 et 3 .

Les méthodes présentées au long de ce papier sont applicables pour construire les maillages associés à différents types de problèmes physiques. Afin d'illustrer quelques réalisations concrètes de domaines traités, nous allons donner des exemples relatifs aux calculs liés à la simulation par Eléments Finis

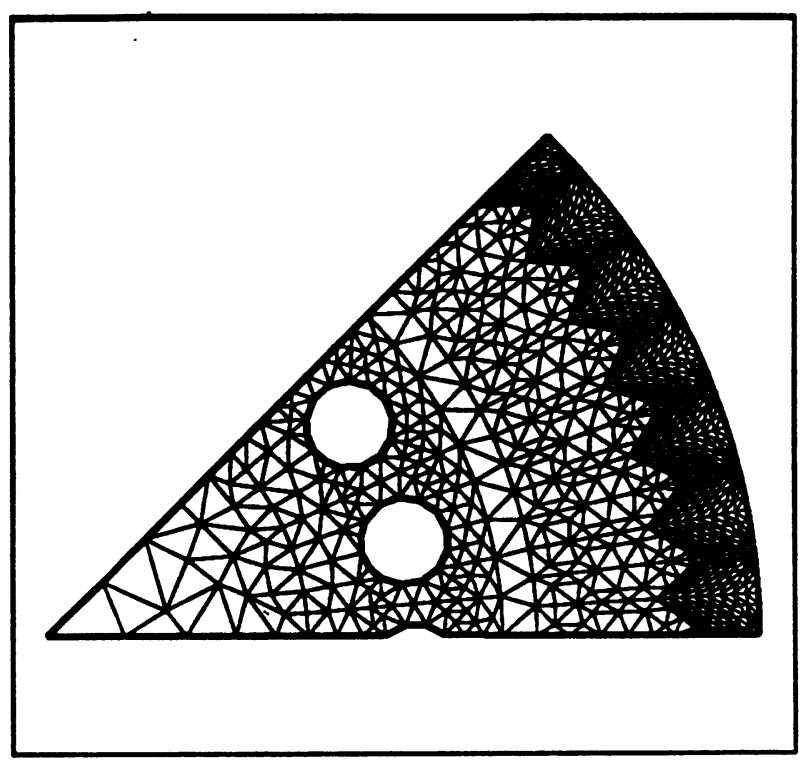

Fig. 13. - Rotor d'un moteur électrique.

[Rotor of an electrical engine.]

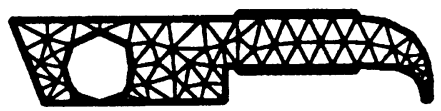

Fig. 14. - Fer et cuivre d'une tête magnétique.

[Iron and copper parts of a magnetic head.] 
intervenant dans le cas de la résolution des problèmes d'électromagnétisme. Il est clair que le champ d'application des mailleurs recouvre bien d'autres domaines [9].

6.1 EXEMPLES EN DIMENSION 2. - Les figures 13 et

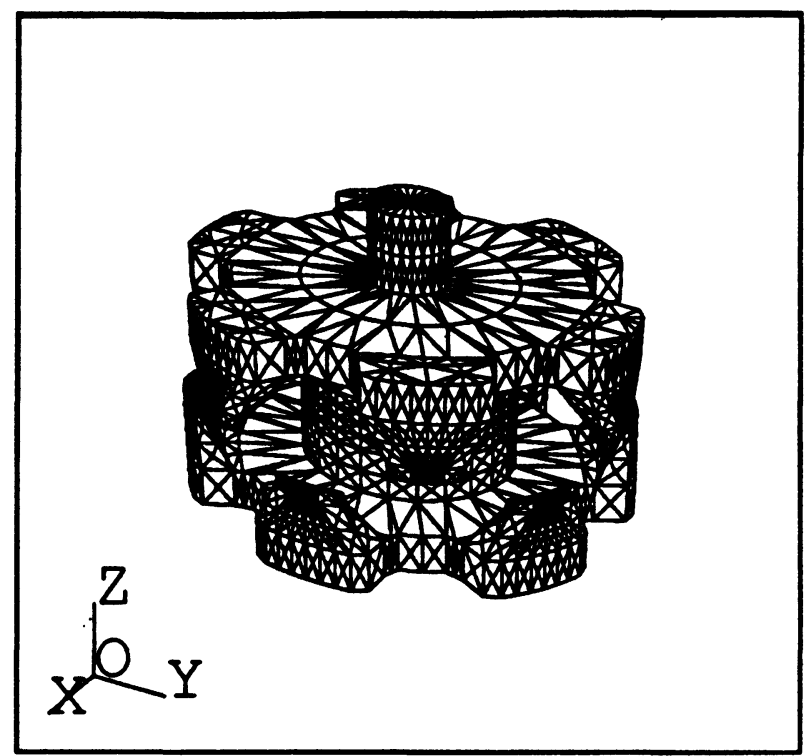

Fig. 15. - Rotor d'un alternateur.

[Rotor of an alternator.]

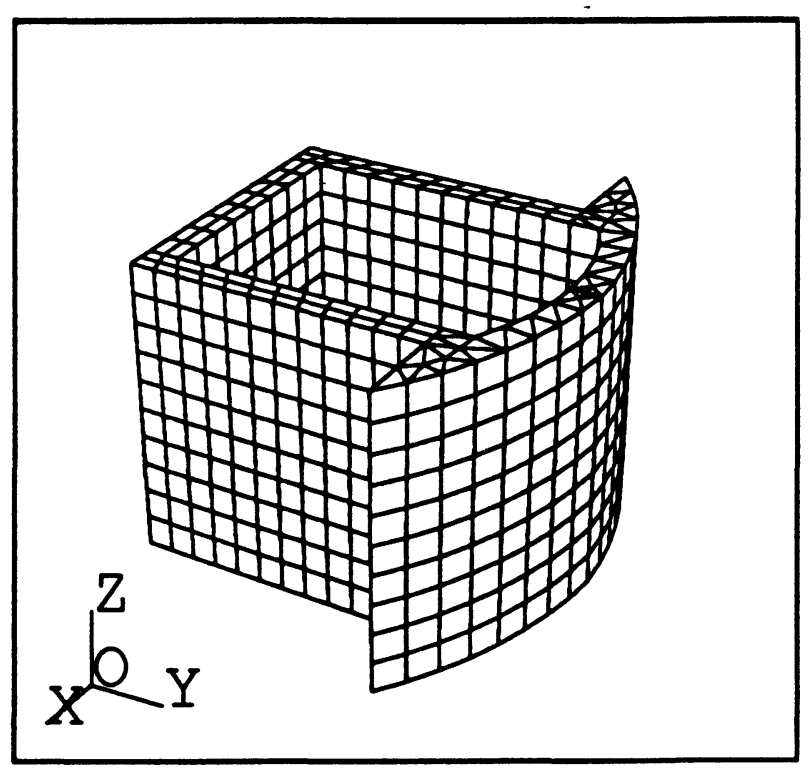

Fig. 16. - Partie d'une tête d'enregistrement.

[Part of a recording head.]

14 montrent deux exemples de géométrie maillée dans le cadre d'une simulation 2D.

6.2 EXEMPLES EN DIMENSION 3. - Les figures 15 et 16 montrent deux exemples de géométrie maillée dans le cadre d'une simulation 3D.

\section{Bibliographie}

[1] BAKER T. J., Generation of tetrahedral meshes around complete aircraft, Numerical grid generation in computational fluid mechanics'88, Miami (1988).

[2] Bernard F., Catia, du dessin au volume, de la cinématique aux calculs scientifiques, de la commande numérique à la robotique, un outil complet de CFAO pour la mécanique, MICAD 84, Hermes (1984).

[3] Cavendish J. C., Automatic triangulation of arbitrary planar domains for the finite element method, Int. J. Num. Meth. Eng. vol. 8 (1974).

[4] Cavendish J. C., Field D. A., Frey W. H., An approach to automatic 3D finite element mesh generation, Int. J. Num. Meth. Eng. vol. 21 (1985).

[5] Cheng J. H., Finnigan P. M., Hathaway A. F., KelA A., SCHOEder W. J., Quadtres/octree meshing with adaptive analysis, Numerical grid generation in computational fluid mechanics'88, Miami (1988).

[6] Ciarlet P. G., The finite element method for elliptic problem (North Holland) 1978.

[7] Coulomb J. L., Maillages 2D et 3D. Expérimentation de la triangulation de Delaunay, Conf. on Automated mesh generation and adaptation, Grenoble (1987).
[8] Coulomb J. L., DU Terrail Y., Meunier G., Flux3d : a finite element package for magnetic computation, Proc. of Compumag'85 (1985).

[9] George P. L., Modulef: Génération automatique de maillage, Collection didactique $n^{\circ} 2$, INRIA (1988).

[10] George P. L., Modulef : Construction et modification de maillages, Rapport Technique $\mathrm{n}^{\circ}$ 104, INRIA (1989).

[11] George P. L., Mailleur 3D par découpage structuré d'éléments grossiers, Rapport de Recherche $n^{\circ}$ 990, INRIA (1989).

[12] George P. L., Utilisation conversationnelle de Modulef, Rapport Modulef $\mathrm{n}^{\circ} 108$, INRIA (1989).

[13] George P. L., La conception descendante appliquée à la réalisation concrète des maillages, Rapport Technique $\mathrm{n}^{\circ}$ 107, INRIA (1989).

[14] George P. L., Golgolab A., Mailleur 3D en topologie "cylindrique ", Rapport Technique $\mathrm{n}^{\circ}$ 100, INRIA (1988).

[15] George P. L., Golgolab A., Muller B., Saltel E., Bibliothèque Modulef : aspects graphiques, Rapport Modulef $\mathrm{n}^{\circ}$ 96, INRIA (1987).

[16] George P. L., Hecht F., Saltel E., Tétraédrisation automatique et respect de la frontière, Rapport de Recherche $n^{\circ} 835$, INRIA (1989). 
[17] George P. L., Hecht F., Saltel E., Maillage automatique de domaines tridimensionnels quelconques, Rapport de Recherche $\mathrm{n}^{\circ}$ 1021, INRIA (1989).

[18] George P. L., Hermeline F., Maillage de Delaunay d'un polyèdre convexe en dimension d'Extension à un polyèdre quelconque, Rapport de Recherche $n^{\circ}$ 969, INRIA (1989).

[19] George P. L. et al., Prétraitement et post-traitement dans le cadre des problèmes d'Eléments Finis, Bulletin de liaison de la recherche en informatique et automatique, INRIA $\mathrm{n}^{\circ} 125$ (1989).

[20] Golgolab A., Mailleur tridimensionnel automatique pour des géométries complexes, Rapport de Recherche $n^{\circ}$ 1004, INRIA (1989).

[21] Hecht F., SAltel E., Emc2 : Editeur de maillages et de contours bidimensionnels, Manuel d'utilisation, Rapport Technique à paraître, INRIA (1989).

[22] Hermeline F., Triangulation automatique d'un polyèdre en dimension $N$, Rairo, Analyse Numérique, vol. 16, $\mathrm{n}^{\circ} 3$ (1982).

[23] Lohner R., PARIKh P., Generation of 3D unstructured grids by advancing front method, AIAA 26 Aerospace Sciences meeting, Reno Nevada (1988).
[24] Modulef et al., Description des Structures de Données, Rapport Modulef $n^{\circ} 2$, INRIA (1989).

[25] Perronnet A., A generator of tetrahedral finite elements for multi-material object and fluids, Numerical grid generation in computational fluid mechanics'88, Miami, 1988.

[26] Shephard M. S., Guerinoni F., Flaherty J. E., Ludwig R. A., BAEHMANN P. L., Finite octree mesh generation for automated adaptive 3D flow analysis, Numerical grid generation in computational fluid mechanics'88, Miami, 1988.

[27] Talon J. Y., Algorithmes d'amélioration de maillages pour éléments finis en 2 et 3 dimensions, Conf. on Automated mesh generation and adaptation, Grenoble, 1987.

[28] Theron M., L'algèbre des solides et la CFAO en mécanique, un exemple: le système Euclid, MICAD 84, Hermes, 1984.

[29] ThOMPSON J. F., Numerical grids generation, North Holland, 1982.

[30] Watson D. F., Computing the $n$-dimensional Delaunay tesselation with applications to Voronoï polytopes, Comput. J. 24 (2) (1981).

[31] Yerri M. A., ShePhARd M. S., Automatic 3D mesh generation by the modified-octree technique, Int. J. Num. Meth. Eng. vol. 20 (1984). 